\title{
Ergonomisch gestaltetes 3-Schichtsystem mit Nachtschichtreduktion für alle Beschäftigten
}

\author{
Corinna JAEGER \\ Institut für angewandte Arbeitswissenschaft e. V. ${ }^{1}$
}

\section{Alternsgerechte Arbeitszeit- und Schichtplangestaltung}

Schichtarbeit - und insbesondere Nachtarbeit - stellt eine besondere Belastung dar, die zu einer erhöhten Beanspruchung führen kann. Nachtarbeit bedeutet, gegen den natürlichen Schlaf-Wach-Rhythmus (Circadian Rhythmic) Leistung erbringen bzw. schlafen zu müssen. Damit steigt das Risiko für Schlafdefizit, Fehler, Unfälle und gesundheitliche Beeinträchtigungen (Jaeger 2015a). Häufige soziale Auswirkungen von Nacht- und Schichtarbeit sind Isolation, eingeschränktes Familienleben und erhöhte Scheidungsrate.

Eine alternsgerechte Arbeitszeit- und Schichtplangestaltung (Jaeger 2014a, 2014b, 2015b) beginnt mit dem Berufseinstieg und erstreckt sich über das gesamte Erwerbsleben. Sie setzt primär auf Prävention, indem Belastung optimiert wird, um vermeidbare Beeinträchtigungen $\mathrm{zu}$ verhindern. Eine Maßnahme besteht darin, Nacht- und Schichtarbeit gemäß arbeitswissenschaftlicher Empfehlungen zu gestalten. Einer der wichtigsten Aspekte ist, Einsätze mit ungünstiger Lage - zum Beispiel Nachtschicht - zu reduzieren (Jaeger 2016).

\section{Praktische Umsetzung in einem Unternehmen der M+E-Industrie}

Am deutschen Standort des Automobilzulieferers arbeiteten 16 Mitarbeiter in einem lang rückwärts rotierten teilkontinuierlichen 3-Schichtsystem mit fünf bis sechs Nachtschichten in Folge. Mehrarbeit wurde ausbezahlt. Das Unternehmen strebte an, zukünftig angemessener und kostengünstiger auf Auftragsschwankungen zu reagieren und die Belastung für die älter werdende Belegschaft zu optimieren. In einem partizipativen Prozess wurde ein flexibles Schichtsystem gemäß arbeitswissenschaftlicher Empfehlungen entwickelt. Bei dem folgenden Beitrag handelt es sich um eine Zusammenfassung der wichtigsten Ergebnisse aus dem Erstartikel der Autorin vom 62. GfA-Frühjahrskongress 2016 (Jaeger 2016)

\footnotetext{
${ }^{1}$ Uerdinger Str. 56, 40474 Düsseldorf. E-Mail: c.jaeger@ifaa-mail.de
} 


\section{Nachtschichtreduktion für alle Beschäftigten}

Im Sinne einer alternsgerechten Arbeitszeitgestaltung realisiert das Unternehmen mit dem ergonomischen Schichtplan (Abbildung 1) folgende arbeitswissenschaftliche Empfehlungen (Beermann 2005).

- Lediglich zwei Nachtschichten in Folge

- Nach Nachtschichtphase 48 Stunden arbeitsfreie Zeit

- Schnelle Rotation von Früh-, Spät- und Nachtschichten

- Vorwärtswechsel der Schichten

- Keine Anhäufung von Arbeitszeiten

- Ungünstige Schichtfolgen vermeiden

- Länge der Schichten den Arbeitsbelastungen anpassen

- Übersichtlicher Schichtplan

- Beschäftigte können ihre Arbeitszeit beeinflussen

Eine Bedarfsermittlung auf Basis von Kennzahlen ergab, dass im Laufe der Woche mehr Teile vorproduziert werden als für die Weiterverarbeitung benötigt. Dieses Ergebnis nutzte das Unternehmen, um im Sinne der Prävention eine nachhaltige Lösung für die Nachtarbeit zu schaffen. An zwei Wochentagen wurden Tätigkeiten aus der Nachtschicht in verlängerte Tagschichten verlagert, wodurch zwei Nachtschichten pro Woche wegfallen. In der dargestellten Hochauslastungsstufe, hat jeder Mitarbeiter lediglich vier Nachtschichten innerhalb des dreiwöchigen Schichtzyklus zu leisten.

Nimmt die Auftragslage ab, entfällt eine weitere Nachtschicht. Auf einem Arbeitszeitkonto verbuchte Plusstunden werden durch Freizeit ausgeglichen - in Absprache mit den Beschäftigten primär durch den Wegfall von Nachtschichten.

Abbildung 1: Ergonomisches 3-Schichtsystems mit Nachtschichtreduktion

\begin{tabular}{|c|c|c|c|c|c|c|c|c|c|c|c|c|c|c|c|c|c|c|c|c|c|}
\hline \multirow{2}{*}{\begin{tabular}{|l|} 
Woche \\
Gruppe \\
\end{tabular}} & \multicolumn{7}{|c|}{1} & \multicolumn{7}{|c|}{2} & \multicolumn{7}{|c|}{3} \\
\hline & Mo & $\mathrm{Di}$ & Mi & Do & $\mathrm{Fr}$ & $\mathrm{Sa}$ & So & Mo & $\mathrm{Di}$ & $\mathrm{Mi}$ & Do & $\mathrm{Fr}$ & $\mathrm{Sa}$ & So & Mo & $\mathrm{Di}$ & Mi & Do & $\mathrm{Fr}$ & $\mathrm{Sa}$ & So \\
\hline A & N & & & $F$ & $F$ & $F$ & & S & $\mathrm{SL}$ & $\mathrm{SL}$ & N & $\mathrm{N}$ & & & $F$ & $F L$ & $F L$ & S & S & & $\mathrm{N}$ \\
\hline B & S & SL & SL & $\mathrm{N}$ & N & & & $\mathrm{F}$ & $\mathrm{FL}$ & $\mathrm{FL}$ & S & S & & N & N & & & $F$ & $F$ & $\mathrm{~F}$ & \\
\hline C & $F$ & $\mathrm{FL}$ & $\mathrm{FL}$ & $\mathrm{S}$ & $\mathrm{S}$ & & $\mathrm{N}$ & $\mathrm{N}$ & & & $F$ & $F$ & $F$ & & S & $\mathrm{SL}$ & SL & N & $\mathrm{N}$ & & \\
\hline & $\mathrm{F}$ & & hich & $\mathrm{hb}$ & & & $\mathrm{S}$ & & hicl & $\mathrm{br}$ & & & N & & & $8 \mathrm{~b}$ & & & & & \\
\hline & $\mathrm{FL}$ & Frü & chich & lang & 5 hbruttc & & SL & & hic & ang & $5 \mathrm{hb}$ & & & & & & & & & & \\
\hline
\end{tabular}

\section{Berücksichtigung von Wünschen der Beschäftigen}

Die 16 Beschäftigten des kleinen Produktionsbereiches wurden in die Gestaltung des neuen Schichtsystems einbezogen. Ihre Arbeitszeitwünsche konnten sie direkt gegenüber dem Betriebsrat bzw. dem Fertigungsleiter äußern, die beide der Projektgruppe angehörten. Die Spätschicht am Samstag war bei den Beschäftigten die unbeliebteste Schicht. Auf ihren Wunsch hin wurde die Betriebszeit von Montag 6:00 Uhr auf Sonntag 22:00 Uhr vorverlegt, sodass sie nun am Samstag nach der Frühschicht endet. Auch den Block mit fünf bis sechs Nachtschichten in Folge empfanden die Beschäftigten als sehr anstrengend. Ihr Wunsch nach kürzeren Nachtschichtphasen wurde ebenfalls berücksichtigt. Zudem war es den Beschäftigten wichtig, 
die eigene Arbeitszeit ein Stück weit beeinflussen und zusätzliche Arbeitszeit durch Freizeit ausgleichen zu können. Dies ist anhand des eingeführten Arbeitszeitkontos möglich. Das kurz vorwärts rotierte Schichtsystem mit unterbrochener Betriebszeit bietet ihnen zudem Spielraum für den Tausch von Schichten.

\section{Pilotierung und Einführung}

Beschäftigte und Betriebsrat standen insbesondere den kurz vorwärts rotierten Schichtfolgen skeptisch gegenüber. Um das gemeinsam entwickelte Schichtsystem anhand konkreter Erfahrungen beurteilen zu können, wurde es sechs Monate lang getestet. Die 16 Beschäftigten tauschten sich über ihre Rückmeldungen direkt mit dem Betriebsrat und dem Fertigungsleiter aus. Die älter werdende Belegschaft spürt eine deutliche Entlastung durch die ergonomischen Schichtfolgen, die geringere Anzahl an Nachtschichten, die kurzen Nachtschichtblöcke und den Ausgleich von Plusstunden durch Freizeit. Auch die Berücksichtigung von Arbeitszeitwünschen der Beschäftigten bei der Entwicklung des Schichtsystems und deren Einfluss auf die eigene Arbeitszeit sorgen für hohe Akzeptanz.

\section{Literaturverzeichnis}

Beermann, Beate und Bundesanstalt für Arbeitsschutz und Arbeitsmedizin (Hg.) (2005) Leitfaden zur Einführung und Gestaltung von Nacht- und Schichtarbeit. 9., unveränderte Auflage. Bundesanstalt für Arbeitsschutz und Arbeitsmedizin, Dortmund

Jaeger, Corinna (2016) Umstellung auf ein ergonomisch gestaltetes 3-Schichtsystem mit Nachtschichtreduktion für alle Beschäftigten. In: Gesellschaft für Arbeitswissenschaft (GfA) (Hg.) Arbeit in komplexen Systemen. Digital, vernetzt, human?! Bericht zum 62. Kongress der Gesellschaft für Arbeitswissenschaft vom 2.-4. März 2016 (Beitrag C 2.4:1-4). Dortmund: GfA-Press

Jaeger, Corinna (2015a) Ergonomische Schichtsysteme als präventive Maßnahme zum Gesundheitsschutz. BPUVZ 127(6):264-267

Jaeger, Corinna (2015b) Ergonomische Arbeitszeitgestaltung - Alternsgerechte Arbeitszeiten. In: Institut für angewandte Arbeitswissenschaft (Hg.) Leistungsfähigkeit im Betrieb. Kompendium für den Betriebspraktiker zur Bewältigung des demografischen Wandels (167-175). Springer, Berlin,

Jaeger, Corinna (2014a) Arbeitszeiten alternsgerecht gestalten. Betriebspraxis \& Arbeitsforschung 220:32-37

Jaeger, Corinna (2014b) Arbeitswissenschaftlich gestaltete Schichtsysteme kommen gut an. Betriebspraxis \& Arbeitsforschung (222):36-41 